\title{
ЭФФЕКТИВНОСТЬ КОМПЛЕКСНОГО ПОДХОДА В ПРОФИЛАКТИКЕ ИНВАЛИДИЗАЦИИ ДЕТСКОГО НАСЕЛЕНИЯ С МИОПИЕЙ НА ПРИМЕРЕ КУРСКОЙ ОБЛАСТИ
}

\author{
(С) Медведева М.В., Ярмамедов Д.М.
}

\author{
Кафедра офтальмологии Курского государственного медицинского университета, Курск \\ E-mail: mari-la2003@mail.ru
}

\begin{abstract}
В статье проанализирована заболеваемость миопией среди детского населения в мире и в России. Оценено количество случаев инвалидности вследствие прогрессирования миопии среди детского населения как в России в целом так и в Курской области. Проведен анализ причин, приводящих к инвалидизации детей из-за осложненной миопии, а также ретроспективный анализ пациентов с прогрессирующей миопией слабой и средней степени в возрасте 8-12 лет. Пациенты были разделены на следующие группы: использующие только очковую коррекцию; дети, которые проходили курсы аппаратного лечения; группа пациентов, которым проводилось аппаратное лечение совместно с использованием медикаментозной терапии. Проведен анализ запаса относительной аккомодации, объема относительной аккомодации и передне-заднего отрезка глаза в течение 12 месяцев. Выявлены наиболее эффективные методы профилактики прогрессирования миопии.
\end{abstract}

Ключевые слова: офтальмология, миопия, инвалидность, лечение, аппаратное лечение, Курск.

\section{ALGORITHM OF WORK OF OPHTHALMOLOGICAL SERVICE ON PREVENTION OF DISABILITY IN CHILDREN WITH MYOPIA BY THE EXAMPLE OF KURSK REGION \\ Medvedeva M.V., Yarmamedov D.M. \\ Department of Ophthalmology of Kursk State Medical University, Kursk}

The article examines the incidence of myopia among children in the world and in Russia. The number of cases of disability due to the progression of myopia among children in Russia and in Kursk region is estimated. The analysis of reasons leading to disability of children due to complicated myopia is carried out. A retrospective analysis of patients with progressive myopia of mild to moderate degree aged 8-12 years has been carried out. Patients were divided into the following groups: those using only a spectacular correction; children who underwent courses of hardware treatment; group of patients who underwent hardware treatment combined with drug therapy. The reserve of relative accommodation, the volume of relative accommodation and the anteroposterior segment of an eye within 12 months have been analyzed. The most effective methods of preventing myopia progression were revealed.

Keywords: ophthalmology, myopia, disability, treatment, hardware treatment, Kursk.

Значительная распространенность заболеваний органа зрения оказывает большое влияние на качество жизни населения. Показатель здоровья является причиной снижения трудоспособности [3]. На данный момент миопия является одной из наиболее актуальных проблем офтальмологии [6].

Количество пациентов с миопией увеличивается с каждым годом [8]. На данный момент около 1,6 млрд. человек во всем мире страдают аномалиями рефракции. 30-45\% взрослого населения России, США, Европы страдают миопией, в ряде районов Восточной Азии этот показатель достигает 70\%. Е.М. Иомдина и Е.П. Тарутта отмечают, что каждый 3-4-й взрослый житель России, США, Европы страдает миопией, и заболеваемость за последние 30 лет выросла в 1,7 раза (с 25 до 45\%), а в урбанизированных районах Восточной Азии достигает 70\% [9].

В России в структуре как первичной, так и вторичной инвалидности среди взрослого населения миопия занимает 3-е место, составив в 2004 г. $-10,6 \%$, в 2005 г. $-16 \%$, в 2012 г. - 23,3\% случаев
$[2,4]$. Проблема инвалидности вследствие миопии актуальна во всем мире, в Индии инвалидизация населения по причине близорукости стоит на втором месте среди причин слепоты [5]. В Азербайджанской республике выявлено, что среди заболеваний органа зрения на инвалидность по поводу миопии приходится 19,6\% $[1,7]$.

В 2016 году в Курской области всего было осмотрено 150890 детей, из них 55\% - школьного и 42\% дошкольного возраста. В 2016 г. заболевания слизистой составили 2890 случаев (рисунок 1). Количество пациентов с миопией составило 1508, данный показатель выше в сравнении с 2015 г. Количество детей с гиперметропией составило 447.

При анализе структуры распределения причин инвалидности детского населения с патологией органа зрения было выявлено, что наиболее распространенной причиной является осложненная миопия. В 2013 г. зафиксировано 53 случая, в 2014 г. - 40, в 2015 г. - 41 случай инвалидности вследствие осложненной миопии. На втором месте по распространенности причин инвалидности 
детского населения атрофии зрительного нерва в 2013 г. зафиксировано 43 случая, в 2014 г. - 32, в $2015-45$.

В связи с ростом количества случаев заболеваемости миопией среди детского населения и высоким процентом инвалидизации как в мире, так и в России и в частности в Курской области, необходим поиск новых комбинированных методов стабилизации миопии, что в конечном итоге снизит количество осложненной миопии, которая ведет к инвалидизации детского и взрослого населения.

\section{МАТЕРИАЛЫ И МЕТОДЫ ИССЛЕДОВАНИЯ}

Проведен ретроспективный анализ 150 (300 глаз) пациентов с прогрессирующей близорукостью слабой и средней степени в возрасте от 8-12 лет, находящихся на диспансерном наблюдении в кабинете охраны зрения, из них - 170 пациентов женского пола и 130 мужского. Срок наблюдения составил 12 месяцев.

Запас относительной аккомодации (3ОА) определяли в условиях полной коррекции для дали с расстояния 33 см в качестве теста был использован текст № 4. Исследование проводили бинокулярно, последовательно располагали отрицательные линзы с шагом 0,5 дптр. Сила максимальной отрицательной линзы определяет положительную (неизрасходованную) часть аккомодации или 3ОА.

Объем относительной аккомодации (ООА) определяли в условиях полной коррекции для дали с расстояния 33 см. ООА вычисляли по арифметической сумме положительной и отрицательной частей относительной аккомодации.

В качестве аппаратного лечения применяли: Визотроник, Витазор (П1-П5), Радуга, АМОАТОС с тауфоном 4\%, электрофорез по Ратнеру с никотиновой кислотой $0,6 \mathrm{MA}-10$ мин.

Было сформировано 3 группы пациентов.

В 1-ю группу вошли пациенты (60), получающие различные виды аккомодотренировок в сочетании с аппаратным лечением 2 курса в год фоне полной очковой коррекции.

Во 2-ю группу (60 пациентов) - получающие различные виды аккомодотренировок в сочетании с аппаратным лечением на фоне полной очковой коррекции миопии и ежедневных инстилляций Фенилефрина $2,5 \%$ на протяжении 1,5 месяца (инстиляции по 1 капли на ночь) 2 курса в год.

В группу контроля вошли 30 пациентов, пользовавшиеся только оптическими методами коррекции.

Учитывая низкую чувствительность методики определения доверительного интервала к типу распределения, а также допустимый для экспериментальных медико-биологических исследований уровень $\mathrm{P} \leq 0,05$, для подтверждения статистической гипотезы был выбран именно такой уровень значимости. Все вычисления выполнялись с помощью аналитического пакета приложения Microsorft Excel Office 2010, лицензией на право использования которой обладает ФГБОУ ВО КГМУ Минздрава РФ.

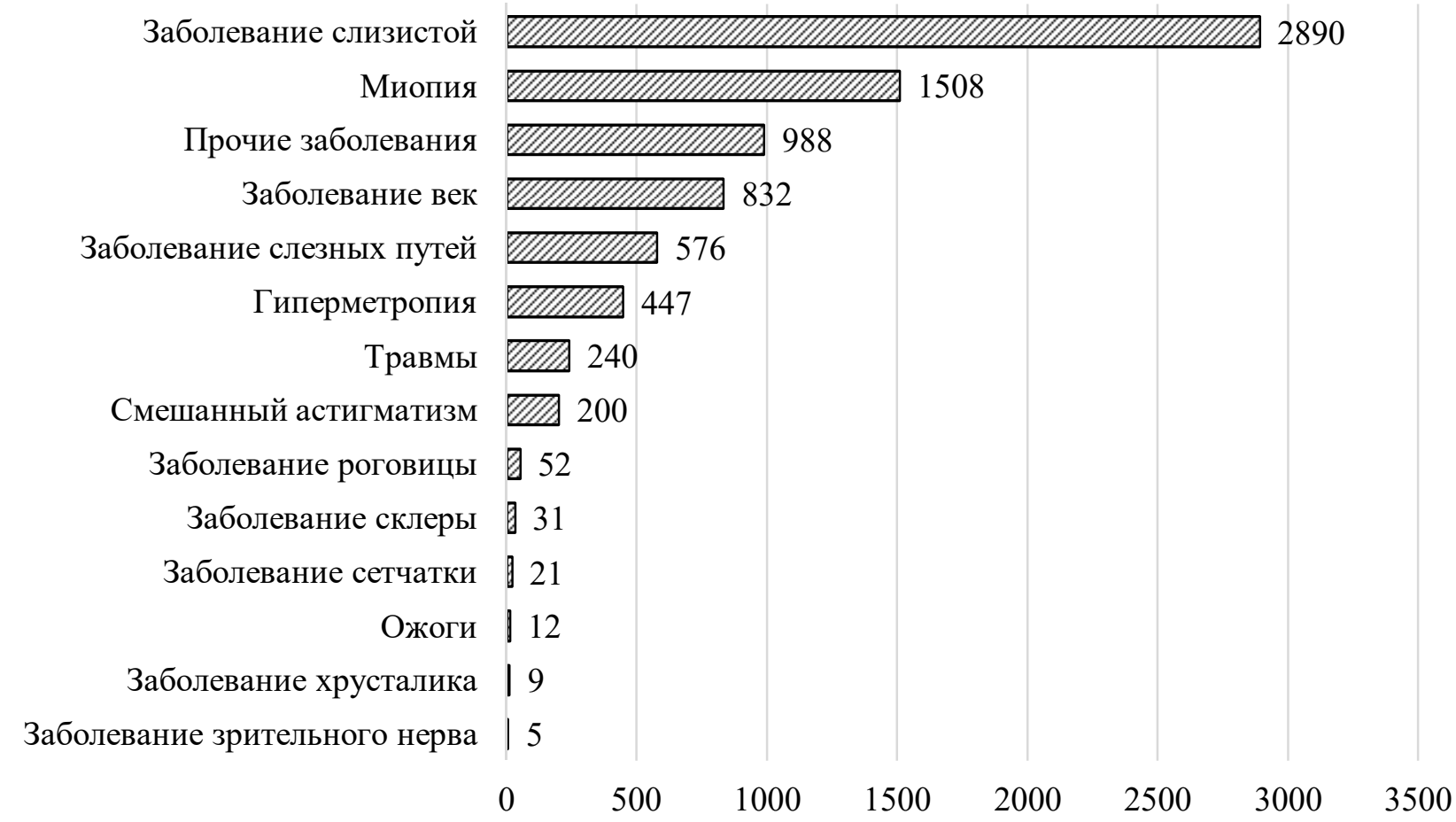

Рис. 1. Структура впервые выявленной офтальмологической патологии в Курской области за 2016 г. 


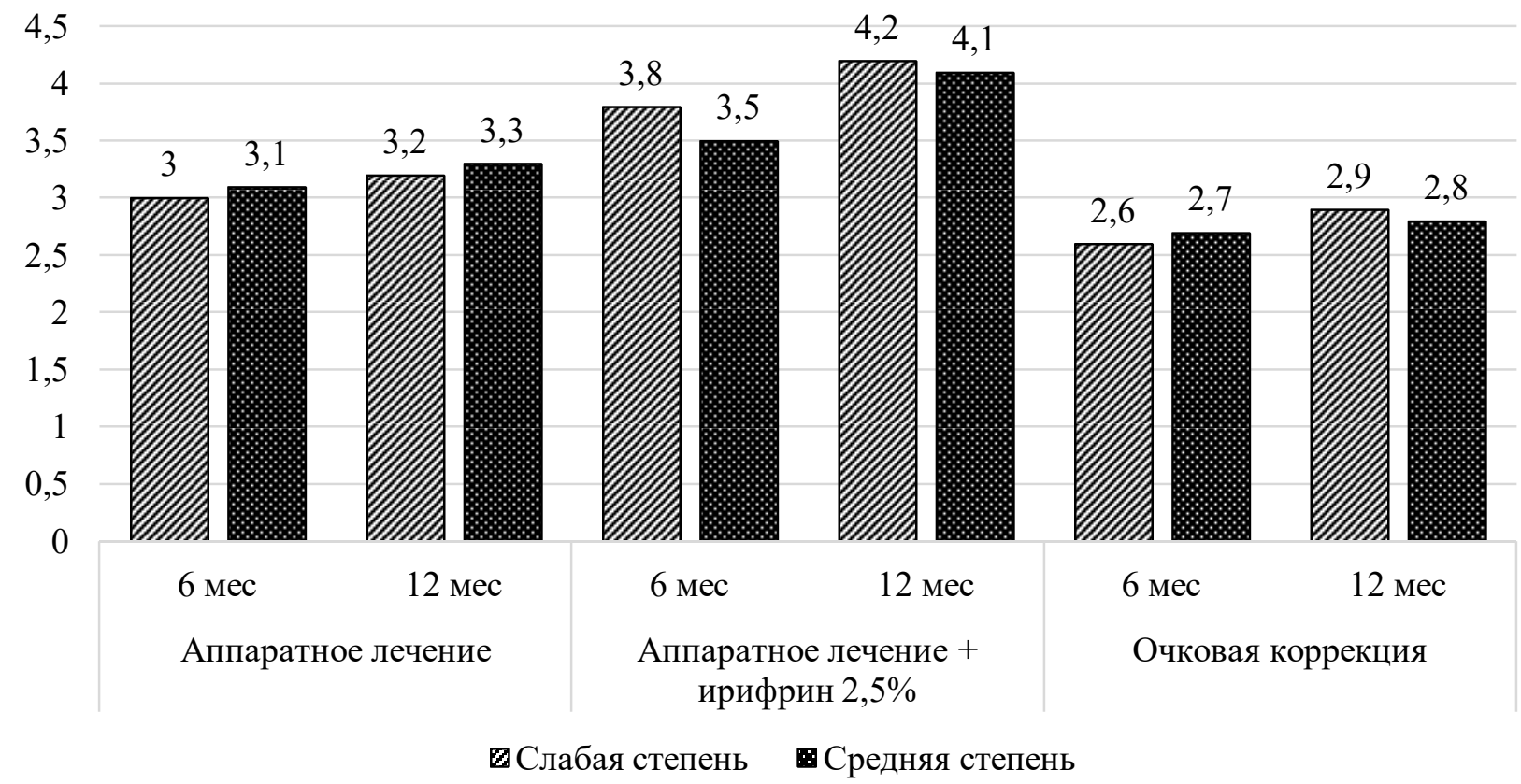

Рис. 2. Динамика запаса относительной аккомодации (3АО, дптр) через 6 мес., 12 мес. (*исследование проводилось в течение 3-х дней после окончания курсов медикаментозного и аппаратного лечения).

\section{РЕЗУЛЬТАТЫ ИССЛЕДОВАНИЯ И ИХ ОБСУЖДЕНИЕ}

При оценке запаса относительной аккомодации (ЗОА) было выявлено, что в группе детей, которые использовали только очковую коррекцию, с миопией слабой степени на сроке наблю-

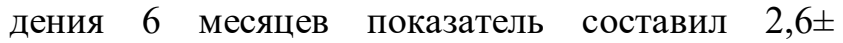

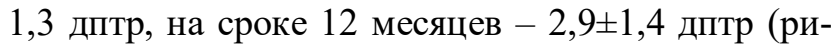
сунок 2). У детей с миопией средней степени на сроке наблюдения 6 мес. $-2,7 \pm 0,76$ дптр, 12 мес. - 2,8 $\pm 0,7$ дптр. При использовании аппаратного лечения у пациентов с миопией слабой степени через 6 месяцев показатель ЗОА составил $3,0 \pm 1,4$ дптр, через 12 месяцев - 3,2 $\pm 0,6$ дптр. У пациентов с миопией средней степени $3,1 \pm 1,4$ дптр на сроке 6 мес, спустя 12 месяцев показатель увеличился на 0,2 дптр. При сочетанном применении аппаратного лечения и медикаментозного лечения выявлено увеличение ЗОА с 3,8 до 4,2 дптр у пациентов с миопией слабой степени. Увеличение показателя на 0,6 дптр (с 3,5 до 4,1 дптр) у детей с миопией средней степени.

При исследовании объема относительной аккомодации в группе с использованием только очковой коррекции на сроке наблюдения 6 месяцев

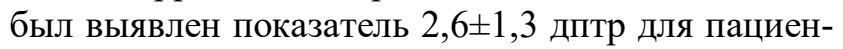

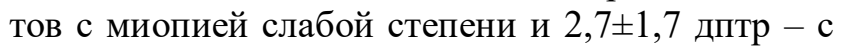
миопией средней степени (рисунок 3 ). При анализе данных в исследуемой группе через 12 месяцев было выявлено незначительное увеличение показателя до 2,9 $\pm 1,6$ дптр в группе с миопией слабой степени и $2,8 \pm 1,7$ дптр в группе с миопией средней степени. У детей с использованием аппаратного лечения на сроке наблюдения 6 месяцев был выявлен показатель ООА равный $6,6 \pm 1,2$ дптр для пациентов с миопией слабой степени и $6,4 \pm 0,8$ дптр для детей с миопией средней степени. При анализе данных через 12 месяцев в исследуемой группе был выявлен показатель $6,4 \pm 1,0$ дптр и $6,8 \pm 1,6$ дптр для пациентов с миопией слабой и средней степени соответственно. Наибольшее увеличение показателя ООА зафиксировано в группе с применением аппаратного лечения в сочетании с медикаментозной терапией - у детей с миопией слабой степени - 0,4 дптр (с 3,8 дптр до 4,2 дптр). У пациентов с миопией средней степени показатель увеличился на 0,6 дптр (с 3,5 дптр до 4,1 дптр).

При анализе полученных результатов было выявлено, что значения 3ОА и ОАА до начала лечения ниже возрастной нормы. В результате проведенного аппаратного лечения показатели повысились у пациентов с разными степенями миопии, что свидетельствует о высокой эффективности проводимого лечения. У пациентов второй группы так же выявлено увеличение 3ОА и OAА, но за более длительный промежуток времени. Таким образом, аппаратное лечение позволяет в короткие сроки улучшить состояние аккомодационного аппарата.

Наиболее важным параметром, характеризующим стабилизацию миопии, является динамика изменения передне-заднего отрезка (ПЗО) глаза (рисунок 4). У пациентов с миопией слабой степени, использующих только очковую коррекцию, выявлена динамика роста на 0,33 мм за 6 месяцев. $\mathrm{У}$ детей с миопией средней степени в данной группе выявлено увеличение ПЗО на 0,35 мм за 6 месяцев. В группе детей с использованием ап- 
паратного лечения рост ПЗО на порядок ниже 0,08 мм и 0,09 мм для пациентов с миопией слабой и средней степени соответственно за 6 месяцев. В группе детей с использованием аппаратного лечения совместно с медикаментозной терапией выявлен рост на 0,03 мм для пациентов с миопией слабой степени и 0,11 мм для пациентов с миопией средний степени в течение 6 месяцев.

По результатам проведенного ретроспективного анализа было выявлено, что наиболее эф- фективным способом лечения детей с миопией является использование аппаратного лечения в сочетании с медикаментозной терапией. Таким образом, оценивая в динамике офтальмопатологию Курской области, была выявлена тенденция к увеличению общей обращаемости к офтальмологам в больницы государственного профиля. При этом выявлен рост заболеваемости по большинству патологий.

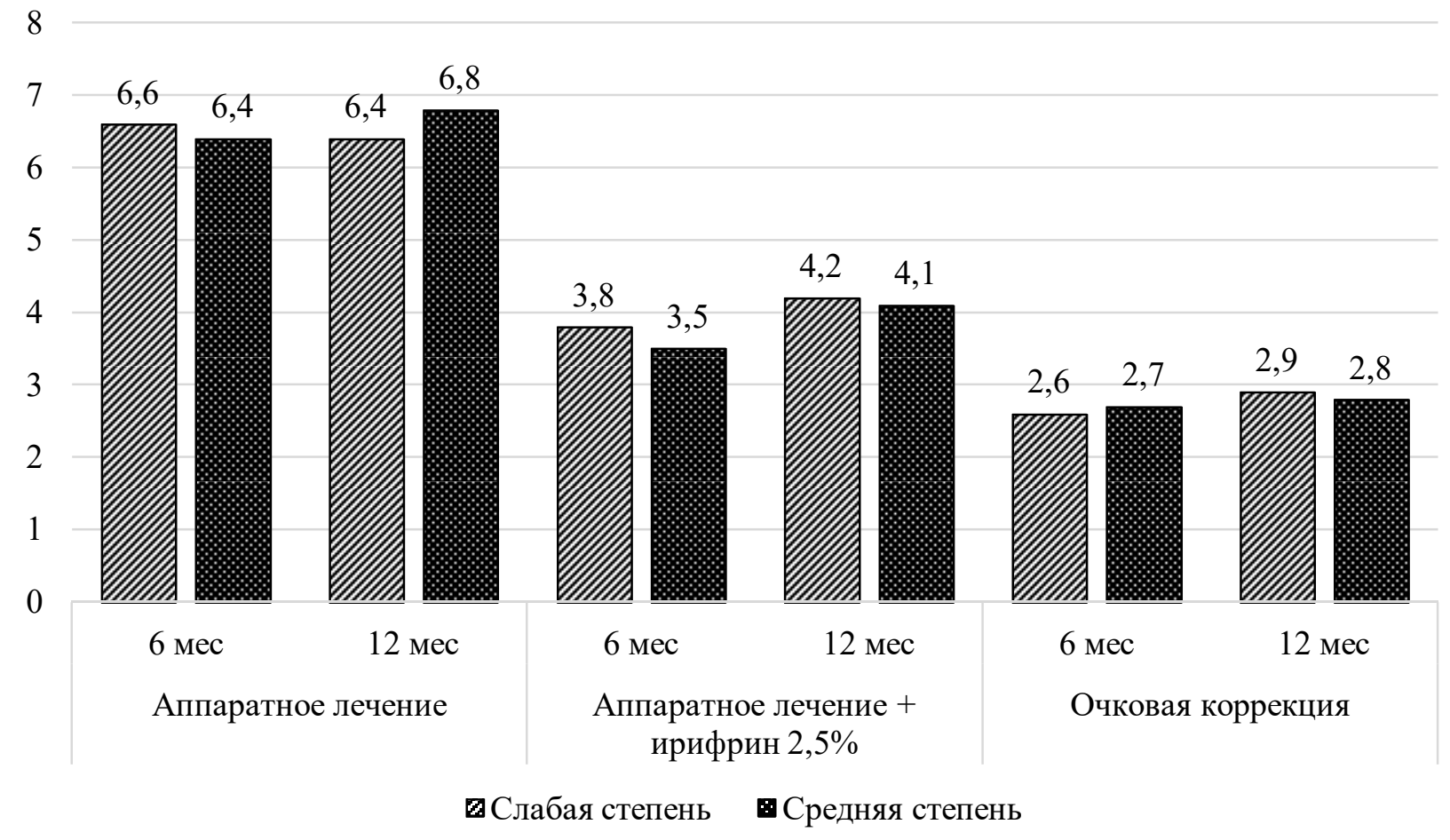

Рис. 3. Динамика объема относительной аккомодации (ОАА, дптр) через 6 мес., 12 мес. (*исследование проводилось в течение 3 дней после окончания курсов медикаментозного и аппаратного лечения).

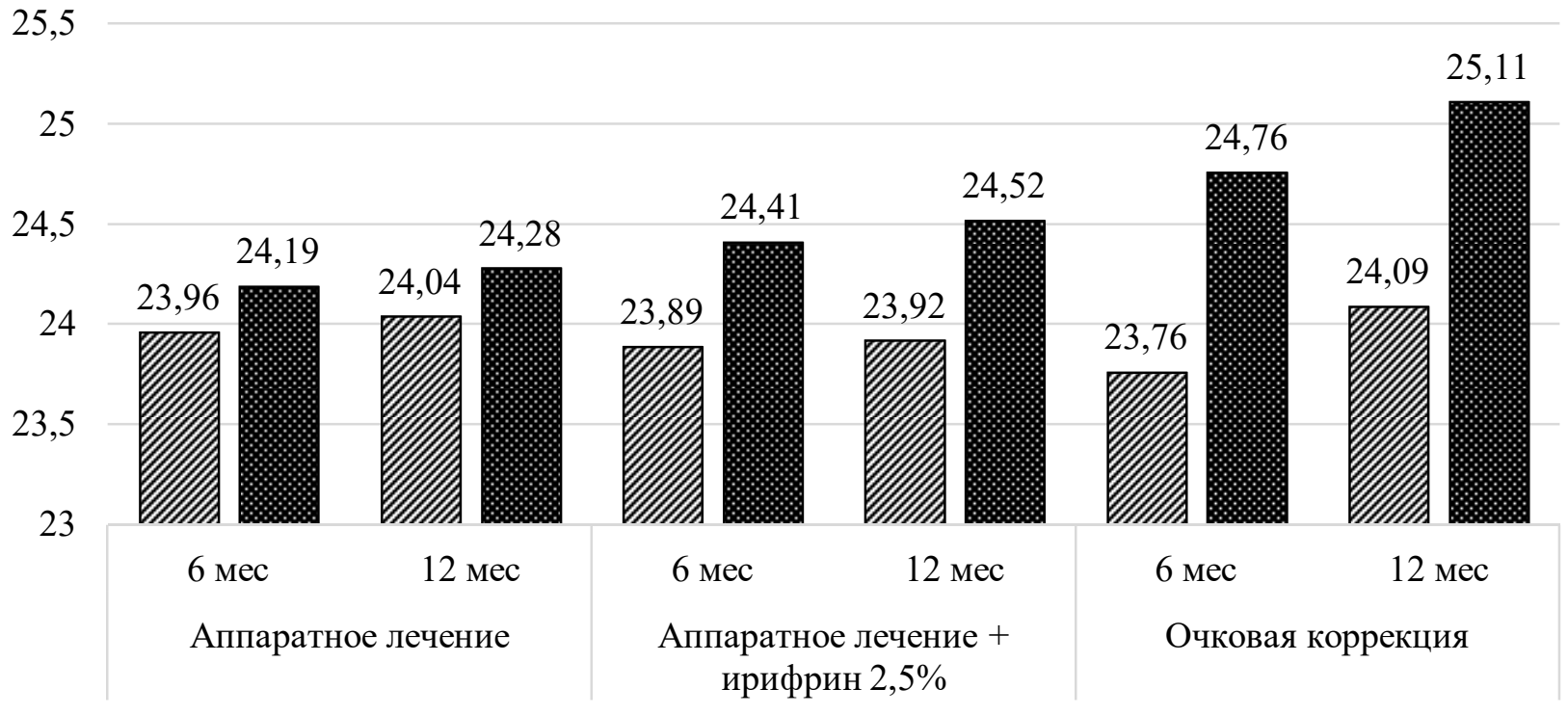

\section{■Слабая степень $\mathbf{0}$ Средняя степень}

Рис. 4. Динамика значений длины ПЗО глаза по данным ОБМ. 


\section{ЛИТЕРАТУРА}

1. Апрелев А.Е., Пашинина Р.В., Караулова Е.С. Оценка распространенности миопии и качества жизни больных с миопией // Медицинский вестник Башкортостана. - 2015. - № 2. - С. 169-171.

2. Баранов В.И., Медведева М.В., Липатов В.А., Новикова А.А., Ярмамедов Д.М. Современные аспекты в лечении инфекционных заболеваний переднего отрезка глаза (обзор литературы) // Электронный научный журнал «Innova». - 2016. - № 2 (3). C. 60-63.

3. Витковская О.П. Стратегия укрепления здоровья в офтальмологии // РМЖ. Клиническая офтальмология. - 2013. - № 3. - С. 88-92.

4. Матросова Ю.В. Сравнительная оценка эффективности различных методов лечения миопии // Вестник Тамбовского университета. Серия: Естественные и технические науки. - 2015. - № 3. C. 642-644.
5. Tарутmа Е.П. Возможности профилактики прогрессирующей и осложненной миопии в свете современных знаний о ее патогенезе // Вестник офтальмологии. - 2006. - Т. 122, № 1. - С. 43-46.

6. Chu $R$. The keypoints of Chinese children myopia prevention and control // Zhonghua Yan Ke Za Zhi. 2014. - Vol. 50, N 1. - P. 6-8.

7. Kedir J., Girma A. Prevalence of Refractive Error and Visual Impairment Among Rural School-Age Children of Goro District, Gurage Zone, Ethiopia // Ethiop J Health Sci. - 2014. - Vol. 24, N 4. - P. 353-358.

8. Saxena R., Vashist P., Menon $V$. Is myopia a public health problem in India? // Indian J Community Med. - 2013. - Vol. 38, N 2. - P. 83-85. - doi: 10.4103/0970-0218.112436.

9. Sun H.P., Li A., Xu Y., Pan C.W. Secular trends of reduced visual acuity from 1985 to 2010 and disease burden projection for 2020 and 2030 among primary and secondary school students in China // JAMA Ophthalmol. - 2015. - Vol. 133, N 3. - P. 262-268. - doi: 10.1001/jamaophthalmol.2014.4899. 